\title{
Encapsulating Peritoneal Sclerosis: A Rare Complication of Peritoneal Dialysis in Al-Ain, United Arab Emirates
}

\author{
Samra Abouchacra ${ }^{\mathrm{a}} \quad$ Ahmed Chaaban $^{\mathrm{a}}$ Suad Sajwani $^{\mathrm{a}}$ \\ Mohammad Bashir $^{\mathrm{b}} \quad$ Omar Beghdash $^{\mathrm{b}} \quad$ Abdishakur Abdulle $^{c}$ \\ Departments of a Nephrology and ${ }^{b}$ Surgery, Tawam-Johns Hopkins Hospital, and \\ ${ }^{\mathrm{C}}$ Department of Internal Medicine, College of Medicine and Health Sciences, United Arab \\ Emirates University, Al-Ain, United Arab Emirates
}

\section{Key Words}

Peritoneal dialysis $\cdot$ Sclerosis $\cdot$ Complications

\begin{abstract}
Encapsulating peritoneal sclerosis (EPS) is a rare complication of peritoneal dialysis (PD) with devastating consequences. Although it can occur among patients on $\mathrm{PD}$, it may present long after PD has been discontinued; thus, its diagnosis depends on a high index of suspicion. We present a rare case of EPS in the United Arab Emirates with a protracted course and delays in recognizing the condition until after resolution of the acute attack. This case highlights the need for early identification of risk factors as well as the subtle features of EPS for appropriate diagnosis and perhaps even prevention; this being worth a ton of cure. A review of the literature including the latest evidence-based treatment options is also highlighted.
\end{abstract}

\section{Introduction}

Encapsulated peritoneal sclerosis (EPS) is a rare and potentially serious complication of long-term peritoneal dialysis (PD) treatment [1, 2]. In 1980, Gandhi et al. [3] reported the earliest description of sclerotic thickening of the peritoneal membrane among patients on long-term (3 months to 4 years) maintenance PD. The authors implicated history of multiple episodes of peritonitis, low $\mathrm{pH}$, and hypertonic dextrose as important contributing factors for the development of EPS. In another study, Denis et al. [4] reported the first case of encapsulated peritonitis unrelated to bacterial infection. The incidence of EPS among 
patients on PD is relatively low and has been estimated at 1.5-4.6 cases per 1,000 patients yearly [5], with significant geographical disparities [6]. Despite its rarity, the timely diagnosis of EPS is important given the associated poor outcome. Preoperative diagnosis depends on a high index of suspicion with attention to subtle, yet characteristic clinical features. We report the first case, to date, in Al-Ain, United Arab Emirates. The diagnosis was not suspected clinically until its complete resolution - months later, emphasizing the need for a better awareness of such a rare entity.

\section{Case Report}

A 40-year-old male Emirati patient with a known history of hypertension, psoriasis and end-stage renal disease, presented to the emergency department because of progressive, generalized, dull abdominal pain with distention, and severe constipation. However, the pain was not radiating to the back and there was no associated vomiting, fever, or diarrhea. The patient's weight and height were $66.7 \mathrm{~kg}$ and $169 \mathrm{~cm}$, respectively. He had been on automated PD prescription (6 cycles, 2-liter exchanges of 1.36 alternating with $3.38 \%$ dextrose) for the past 6 months. Previously, he had been maintained on chronic ambulatory peritoneal dialysis 2 -liter exchanges of $1.5 \%$ dextrose for a number of years. Recently, he had evidence of ultra-filtration membrane failure with declining drain volumes, hence, the change to automated PD, after which he showed good improvement. Prior to hospitalization, his spouse noticed turbidity in the PD fluid, but no other problems were reported.

Past clinical history was significant for end-stage renal disease of unknown etiology for over 20 years and hypertension. He had a living related renal transplantation from his father. Unfortunately, he suffered allograft rejection within 3 months of transplantation. Subsequently, he underwent allograft nephrectomy and was on maintenance hemodialysis for 13 years. Later, he switched to PD modality which has been ongoing for 8 years. He denied prior history of peritonitis. His current medications include: nifedipine $60 \mathrm{mg}$ OD, atenolol $25 \mathrm{mg} \mathrm{OD}, \mathrm{CaCO}_{3} 1.2 \mathrm{~g}$ TID, and omeprazole $20 \mathrm{mg}$ OD.

Physical examination revealed a mildly distressed, unwell looking gentleman who was afebrile $\left(36.8^{\circ} \mathrm{C}\right)$, blood pressure $144 / 86 \mathrm{~mm} \mathrm{Hg}$, pulse $72 / \mathrm{min}$, and respiratory rate 18 breaths/min. Cardiovascular examination was unremarkable with normal heart sounds, regular rhythm, and no murmur. Peripheral pulses were palpable with no edema. Lungs were clear and abdominal examination showed a distended abdomen with left lower quadrant tenderness and rebound, but normal bowel sounds. There was evidence of a previous surgical scar and the PD exit site had no redness or discharge.

\section{Laboratory Investigations}

Laboratory investigations revealed a white blood cell count of $11,000 \times 10^{9} / 1$ with $85 \%$ neutrophils and $8.2 \%$ lymphocytes. Serum C-reactive protein was high at $344 \mathrm{mg} / \mathrm{l}$, serum sodium: $135 \mathrm{mmol} / \mathrm{l}$, potassium: $4.1 \mathrm{mmol} / \mathrm{l}$, chloride: $94 \mathrm{mmol} / \mathrm{l}$, bicarbonate: $26 \mathrm{mmol} / \mathrm{l}$, and glucose: $6.4 \mathrm{mmol} / \mathrm{l}$; all levels were within normal limits. Blood urea nitrogen was 21 $\mathrm{mmol} / \mathrm{l}$ and creatinine $1,540 \mu \mathrm{mol} / \mathrm{l}$. Liver function tests were normal. Also, chest X-ray did not show any significant abnormalities. Abdominal ultrasound showed a mixed echogenicity fluid collection in the pelvis. The PD catheter was in place without any other intra-abdominal abnormalities. Abdominal CT scan showed evidence of peritonitis with paralytic ileus and a thickened wall of the sigmoid colon (fig. 1). No cytology of the dialysate was available. 


\section{Hospital Course}

The patient was started on cefazolin ( $1 \mathrm{~g} / \mathrm{IP}$ daily) and gentamicin ( $40 \mathrm{mg} / \mathrm{IP}$ daily) as per ISPD guidelines 2000 [7]. He continued to exhibit temperature spikes during his first 2 days in hospital with continued abdominal pain and turbid PD fluid. On day 5, the surgical team, who had been monitoring the patient since admission, elected to perform an exploratory laparotomy and remove the PD catheter. The procedure revealed inflamed bowel loops with cocooning (fig. 2). The patient was then switched to hemodialysis via left AV fistula. However, despite removing the PD catheter, he remained febrile with continued abdominal pain and distention. Serum C-reactive protein level remained $>200 \mathrm{mg} / \mathrm{l}$. After consultation with infectious disease physicians, he was switched to meropenem $1 \mathrm{~g} /$ day following hemodialysis. Nonetheless, his fever and abdominal distention persisted unabated for over 3 months. He further underwent multiple treatment courses of antibiotics and antifungal agents including piperacillin-tazobactam, meropenem, tigecycline, voriconazole, caspofungin, metronidazole, vancomycin, and levofloxacin with no significant improvement. Throughout the course of hospitalization, he had intermittent loose stools. Moreover, he was marginally tolerating oral intake and required nutritional supplementation. Overall, his general condition deteriorated during his hospital stay, he lost approximately $10 \%$ of his body weight, and his serum albumin decreased to $32 \mathrm{mmol} / \mathrm{l}$. Of note is that the culture of various specimens including blood, PD fluid, stool, and clostridium difficile antigen and toxin remained negative throughout his hospitalization.

Entertaining a wide spectrum of differential diagnoses to include tuberculous peritonitis, autoimmune disease, and familial Mediterranean fever, the following investigations were performed: QuantiFERON-TB and other serological investigations, namely ANA, ANCA, C3, C4, CMV, hepatitis B, C and HIV, were also negative. After removal of the PD catheter, the wound was healing well and wound cultures were negative with the exception of one which grew non-albicans Candida, hence, the antifungal treatment. Repeat abdominal ultrasound showed no major changes since admission. He then underwent a trial of discontinuing all antibiotics. Re-culture analyses were carried out, but remained negative. Subsequently, the patient was prescribed a trial anti-tuberculosis treatment regimen consisting of isoniazid (300 mg/day), rifampicin (600 mg/day), moxifloxacin ( $400 \mathrm{mg} /$ day), ethambutol (1,200 mg post HD) and pyridoxine (50 mg/day). He was discharged home (4.5 months later) to continue on thrice weekly HD.

However, due to intolerance, he stopped anti-tuberculosis medications 2 weeks after discharge. Despite not taking his medication regularly, his condition slowly improved, and he became afebrile after 4 weeks with complete resolution of all his symptoms. It was at this juncture that the diagnosis of EPS was entertained in retrospect. Since then, the patient has had two similar presentations with abdominal pain secondary to subacute small bowel obstructions that responded well to supportive treatment with hydration, Nasogastric drainage, and pain control.

\section{Discussion}

EPS, though uncommon, is a potentially lethal complication of PD with unclear pathogenesis. Known risk factors for EPS include long duration of PD, exposure to high glucose concentrations in dialysis solution, and severe bacterial peritonitis. Also, a decline in ultrafiltration in high transporters is an early indicator of EPS development. Our patient demonstrated high-risk characteristics for EPS, with long exposure to maintenance PD for almost 8 years, and a history of using high glucose concentration in dialysate in the recent 
past but, interestingly, with no prior history of peritonitis. In addition, the patient had evidence of ultrafiltration failure prior to his presentation with the need to change to automated PD and the use of hypertonic PD fluid. More importantly, our patient had atypical clinical features of EPS including, but not limited to, abdominal pain, nausea, vomiting, and intestinal obstruction. Taken together, our patient appears to have had several known risk factors, all of which should have led to a timely diagnosis and better treatment outcome.

EPS is characterized by a progressive intra-abdominal inflammation resulting in sheets of fibrous tissue that encapsulate, bind, and constrict the viscera, thus restricting bowel movement and function. The hallmark pathological features of EPS include peritoneal thickening and calcification, loculated ascites, narrowing bowel lumen with adherent loops, and encapsulation by fibrous cocoon. Laboratory findings include elevated C-reactive protein and $\mathrm{TGF}_{\beta 1}$ as well as decreased hemoglobin, serum albumin, and CA-125 levels. CT scan is also the most helpful tool, though less definitive than laparoscopy and biopsy [8]. Overall, three out of the following criteria are diagnostic of EPS, namely peritoneal enhancement, thickening, calcifications, bowel tethering, dilatation, and fluid loculation/septation [9].

When reviewed in retrospect, our patient possessed four of the above characteristic features on CT scan as shown in fig. 2. Nonetheless, there was a significant delay in diagnosing EPS in this patient; in fact, the case was diagnosed once the acute phase had completely resolved. The patient was thus exposed to prolonged and multiple courses of antibiotics rather than appropriately targeted treatment.

Current therapy for EPS focuses on the use of immune suppressive and antifibrotic agents such as tamoxifen, prednisone, azathioprine, or mycophenolate mofetil [10-13]. However, the data on the latter two is weak, whereas tamoxifen and steroids are advocated as useful in prevention as well as treatment of EPS. Experimental data suggest that rosiglitazone may also be useful in halting the progression and even causing regression of EPS [14]. N-acetylcysteine has been shown to decrease inflammation and vascularity of PD membranes. Similarly, colchicine has been shown to have anti-inflammatory protective effects. RAAS blockade appears to reduce cell proliferation and fibrosis, which can prevent EPS progression and might play a role in its prevention through improvement of high transporter characteristics and UF failure. Other agents with a potential protective effect include thalidomide, everolimus and a number of other experimental agents. Biocompatible PD solutions have long been proposed as protective measures. However, there are no randomized trials to clearly establish their utility in the prevention of EPS.

To date, no definitive surgical treatment for EPS has been characterized due to limited clinical experience. Often times, conservative measures may obviate the need for surgical interventions. However, when the latter is needed, complete enterolysis of intestinal adhesions without enterectomy has shown a successful improvement of up to 94.2\% [15]. Recent data suggest that perionectomy and enterolysis (PEEL), though a complex procedure, is a promising treatment option that may be performed with low mortality and acceptable morbidity [16].

It should be noted that whilst surgery can reverse intestinal obstruction, it does not improve peritoneal deterioration; thus, such procedures, though relatively successful, may lead to heightened recurrence rates of EPS [15]. Undoubtedly, specific risk factors must be recognized early enough, especially among asymptomatic patients to avoid complications.

Such measures include avoidance of long durations of PD ( $>7$ years) and continuous assessment of PET to detect occurrence of high transporter characteristics. Furthermore, the use of biocompatible dialysis solution has been advocated to reduce the occurrence of EPS. Other measures include reducing glucose load and preventing peritonitis. PD rest or 'expiry 
date', implying PD discontinuation according to a pre-specified time, even before EPS is suspected, has been suggested. Such an approach, however, was recently challenged by Cornelis and Oreopoulos [2], who proposed the development of an EPS scoring system based on clinical, laboratory, and radiological features along with preventative treatment, namely tamoxifen and/or prednisone at a specified threshold. Randomized trials are necessary to validate this strategy.

\section{Conclusion}

EPS is a rare complication of PD that may lead to devastating clinical consequences. Though it can occur in patients on PD, it may present long after PD has been discontinued, such as in the posttransplant period. Hence, EPS diagnosis depends on a high index of suspicion coupled with several laboratory and radiological tests. Most importantly, early identification and recognition of its subtle features is key for prevention.

\section{References}

1 Kawaguchi Y, Kawanishi H, Mujais S, Topley N, Oreopoulos DG: Encapsulating peritoneal sclerosis: definition, etiology, diagnosis, and treatment. International Society for Peritoneal Dialysis Ad Hoc Committee on Ultrafiltration Management in Peritoneal Dialysis. Perit Dial Int 2000;20:S43-S55.

-2 Cornelis T, Oreopoulos DG: Update on potential medical treatments for encapsulating peritoneal sclerosis; human and experimental data. Int Urol Nephrol 2011;43:147-156.

-3 Gandhi VC, Humayun HM, Ing TS, Daugirdas JT, Jablokow VR, Iwatsuki S, et al: Sclerotic thickening of the peritoneal membrane in maintenance peritoneal dialysis patients. Arch Intern Med 1980;140:1201-1203.

-4 Denis J, Paineau J, Potel G, Fontenaille C, Guenel J: Continuous ambulatory peritoneal dialysis. Ann Intern Med 1980;93:508.

5 Yokota S, Kumano K, Sakai T: Prognosis for patients with sclerosing encapsulating peritonitis following CAPD. Adv Perit Dial 1997;13:221-223.

6 Saito A: Peritoneal dialysis in Japan: the issue of encapsulating peritoneal sclerosis and future challenges. Perit Dial Int 2005;25:S77-S82.

7 Afthentopoulos IE, Passadakis P, Oreopoulos DG, Bargman J: Sclerosing peritonitis in continuous ambulatory peritoneal dialysis patients: one center's experience and review of the literature. Adv Ren Replace Ther 1998;5:157-167.

8 Nakayama M: The plasma leak-to-response hypothesis: a working hypothesis on the pathogenesis of encapsulating peritoneal sclerosis after long-term peritoneal dialysis treatment. Perit Dial Int 2005;25:S71S76.

9 Vlijm A, Stoker J, Bipat S, Spijkerboer AM, Phoa SS, Maes R, et al: Computed tomographic findings characteristic for encapsulating peritoneal sclerosis: a case-control study. Perit Dial Int 2009;29:517-522.

10 Mori Y, Matsuo S, Sutoh H, Toriyama T, Kawahara H, Hotta N: A case of a dialysis patient with sclerosing peritonitis successfully treated with corticosteroid therapy alone. Am J Kidney Dis 1997;30:275-278.

11 Diaz-Buxo JA: Peritoneal sclerosis in a woman on continuous cyclic peritoneal dialysis. Semin Dial 1992;5:317-320.

12 del Peso G, Bajo MA, Gil F, Aguilera A, Ros S, Costero O, et al: Clinical experience with tamoxifen in peritoneal fibrosing syndromes. Adv Perit Dial 2003;19:32-35.

$\checkmark 13$ Eltoum MA, Wright S, Atchley J, Mason JC: Four consecutive cases of peritoneal dialysis-related encapsulating sclerosis treated successfully with tamoxifen. Perit Dial Int 2006;26:203-206.

14 Bozkurt D, Taskin H, Sezak M, Biçak S, Sen S, Ok E: Duman S. Rosiglitazone, a peroxisome proliferatoractivated receptor agonist, improves peritoneal alterations resulting from an encapsulated peritoneal sclerosis model. Adv Perit Dial 2008;24:32-38.

15 Kawaguchi Y, Saito A, Kawanishi H, Nakayama M, Miyazaki M, Nakamoto H, et al: Recommendations on the management of encapsulating peritoneal sclerosis in Japan, 2005: diagnosis, predictive markers, treatment, and preventive measures. Perit Dial Int 2005;25:S83-S95.

16 Ulmer C, Braun N, Rieber F, Latus J, Hirschburger S, Emmel J, et al: Efficacy and morbidity of surgical therapy in late-stage encapsulating peritoneal sclerosis. Surgery 2013;153:219-224. 
Case Reports in

Nephrology and Urology

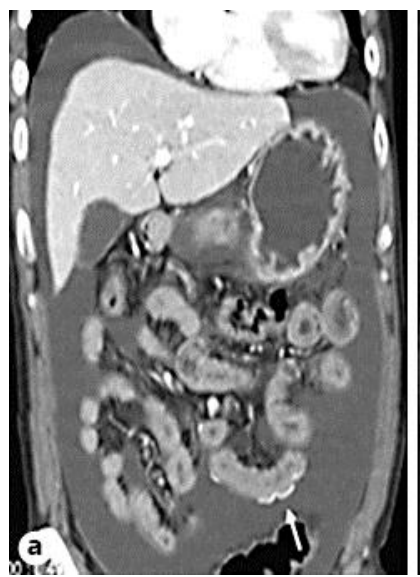

Abouchacra et al: Encapsulating Peritoneal Sclerosis: A Rare Complication of Peritoneal Dialysis in Al-Ain, United Arab Emirates

Fig. 1. Plain abdominal CT scan taken after admission showing evidence of peritonitis with paralytic ileus and thickened wall of the sigmoid colon. a Peritonitis with paralytic ileus, thickened wall of the sigmoid colon, and peritoneal enhancement. b Bowel tethering and dilatation with fluid loculation. Asterisk indicates massively dilated transverse colon.

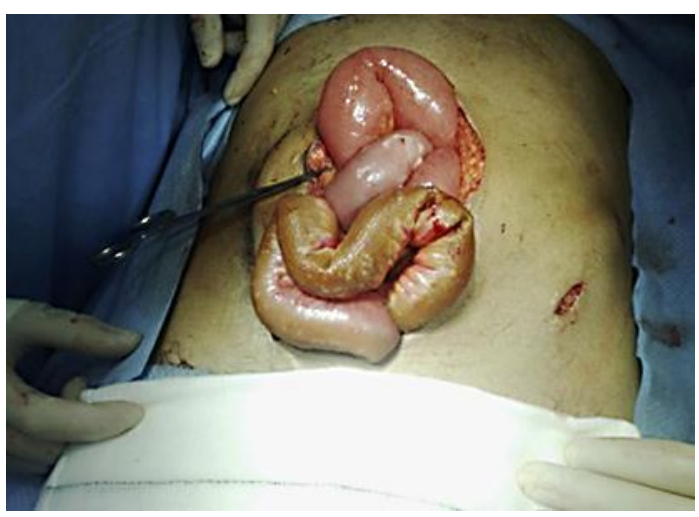

Fig. 2. The sigmoid colon and bowel found in the pelvis (in contact with the PD catheter) show thickening. The serosa appears rough (tethered) and snake-like in texture and light brown in color. 